\title{
Sniffing methanol in hand sanitizers
}

$30 *$ Corresponding author: andreas.guentner@ptl.mavt.ethz.ch 


\section{Abstract}

32 The COVID-19 pandemic has increased dramatically the demand for hand sanitizers. A major

33 concern is their adulteration with methanol that caused more than 700 fatalities in Iran and

34 U.S.A. (since Feb. 2020). In response, the U.S. Food and Drug Administration (FDA) has

35 restricted the methanol content in hand sanitizers to 0.063 vol\% and blacklisted 194 products (as

36 of Oct. 1, 2020). Here, we present a low-cost, handheld and smartphone-assisted device that

37 detects methanol selectively in hand sanitizers between $0.01-100$ vol\% within two minutes by

38 headspace analysis. It features a nanoporous polymer column that separates methanol from

39 confounders by adsorption (i.e. van-der-Waals forces) rendering it selective. A chemoresistive

40 gas sensor detects the methanol. When tested on seven pure and spiked commercial sanitizers

41 (total 76 samples), methanol was quantified accurately, in excellent $\left(\mathrm{R}^{2}=0.99\right)$ agreement to

42 "gold standard" gas chromatography. Most importantly, methanol quantification was hardly

43 interfered by different sanitizer compositions (e.g. 2-propanol, ethanol, butanone, glycerin, aloe

44 vera essence, various odorants and colorants) and gel-like viscosity while other potential

45 contaminants (e.g. 1-propanol) were recognized as well. This device meets an urgent need for

46 distributed and on-site methanol screening by authorities (e.g. customs, police), health product

47 distributers and even laymen.

48 Keywords:

49 Public health, hazardous material monitoring, disinfectant, chemical detection, SARS-CoV-2. 


\section{Introduction}

51 The global health emergency due to the infectious respiratory disease SARS-CoV-2 or COVID-

5219 (Wu et al., 2020) has caused a rapid increase in hand sanitizer consumption that led

53 temporarily to acute shortages in supply. In response, global production has grown involving

54 also small businesses (e.g. distilleries) and universities (Dicken et al., 2020) that produce and

55 distribute hand sanitizers often locally at small scale. Public awareness about safety issues in

56 hand sanitizers has emerged since the FDA placed a warning for 194 products (by Oct. 1, 2020)

57 (U.S. Food and Drug Administration, 2020a) that contained up to 81 vol\% of toxic methanol,

58 drastically exceeding recommended (U.S. Food and Drug Administration, 2020b) limits (0.063

59 vol\%). Similar hand sanitizer concerns have been published by the Canadian government

60 (Government of Canada, 2020). The ingestion of methanol-contaminated sanitizers led already to

61 more than 700 fatalities in Iran (Wambua-Soi, 2020) and the U.S.A. (Fazio, 2020) since Feb.

622020.

63 Commercial hand sanitizers should contain only ethanol or 2-propanol for antisepsis,

64 according to the World Health Organization (WHO) (World Health Organization, 2010). For

65 instance, after 30 seconds, the viral infectivity of SARS-CoV was reduced by more than 4 or 3

66 orders of magnitude with $80 \mathrm{vol} \%$ ethanol or $70 \mathrm{vol} \%$ 2-propanol, respectively. (Kampf et al.,

67 2020) Other substances like glycerol (humectant), hydrogen peroxide (against bacterial spores),

68 odorants and colorants may be contained as well (World Health Organization, 2010). Methanol

69 is colorless and hardly distinguishable by odor from other alcohols like ethanol, so it cannot be

70 recognized easily by human olfaction or vision. Its toxicity is primarily related to its metabolic

71 products formaldehyde and formic acid (Barceloux et al., 2002) that can cause permanent

72 neurologic dysfunctions, ocular morbidity up to blindness or even death (Kraut and Mullins, 
73 2018). Therefore, low-cost and portable methanol detectors are needed to assist distributors,

74 local authorities and even consumers to check product safety. Analytically challenging for such

75 detectors are the required selectivity over other hand sanitizer ingredients, the large methanol

76 detection range (at least $0.063-81 \mathrm{vol} \%$ ), fast response times and, ideally, repeated usability.

77 Gas or liquid chromatography are most established for methanol detection in complex

78 mixtures, but these are bulky, expensive instruments that require trained personnel, (Kraut and

79 Kurtz, 2008) usually available only in specialized laboratories and unsuitable for on-site

80 analyses. (Kraut and Mullins, 2018) Also optical infrared detectors suffer from similar

81 drawbacks, for instance, the DX4000/DX4015 (Gasmet Technologies) that weighs $15 \mathrm{~kg}$ and is

82 rather expensive (tens of thousands of US\$ (U.S. Department of Defense, 2020)). Cheaper (Park

83 et al., 2020), more compact (Weber et al., 2020) and less power consuming (Güntner et al., 2020)

84 are chemical gas sensors (e.g. Pt-loaded tungsten nitride (Meng et al., 2020), polymer-coated Si

85 bridges (Guo et al., 2011), electrochemical cells ( $\mathrm{Ou}$ et al., 2019) or nanoporous $\mathrm{Al}_{2} \mathrm{O}_{3}$-coated

86 carbon nanotubes (Zhao et al., 2012)) that detect methanol from the headspace of liquids.

87 However, most are interfered by ethanol that is usually present at high content (Table 1) and

88 none has been tested on hand sanitizers. Finally, a colorimetric assay (Alert for Methanol,

89 Neogen Corp., ca. \$20 per test) is available for alcoholic beverage analysis, which indicates if

90 methanol is below or above $0.35 \mathrm{vol} \%$, but is insufficient to check FDA adherence. Also, it is

91 single-use, requires cooling $\left(2-8^{\circ} \mathrm{C}\right)$ and might be interfered particularly by colorants but also

92 other hand sanitizer ingredients (e.g. 2-propanol, glycerol, odorants) and may fail on gel-like

93 hand sanitizers.

94 Here, we present an inexpensive and compact device that quantifies hazardous methanol

95 accurately in hand sanitizers by headspace analysis. It comprises a separation column of Tenax 
96 TA particles and a chemoresistive gas sensor of Pd-doped $\mathrm{SnO}_{2}$ nanoparticles (van den Broek et

97 al., 2019) integrated into a smartphone-assisted analyzer with validated performance for

98 alcoholic drinks (Abegg et al., 2020). Here, we applied it to seven pure and methanol-spiked

99 (0.01-90 vol\%) commercial hand sanitizers (total 76 samples) with various compositions

100 (Table 1) to assess its resistance to challenging 2-propanol, glycerol, various odorants and gel-

101 like viscosity. Results were compared to established gas chromatography as recommended by

102 FDA (U.S. Food and Drug Administration, 2020b).

103 Materials and methods

104 Device design

105 The handheld detector is shown in Figure 1 and its design elaborated elsewhere (Abegg, et al.,

106 2020). In brief, vapor from the headspace of liquid samples was extracted with a capillary

107 (Sterican, B. Braun, Germany) fixed to a Teflon tube (4 $\mathrm{mm}$ inner diameter). This tube contained

108 the sorption material, $150 \mathrm{mg}$ Tenax TA powder (60-80 mesh, $\sim 35 \mathrm{~m}^{2} \mathrm{~g}^{-1}$, poly(2,6-diphenyl-p-

109 phenylene oxide), Sigma Aldrich, Switzerland) (van den Broek, et al., 2019), that was fixed as

110 packed bed with tension springs and silanized glass wool plugs to avoid voids. Note that such

111 separation columns could be miniaturized even further by microfabrication and their loading can

112 be varied flexibly to adjust analyte separation for other analytes (e.g. formaldehyde (van den

113 Broek et al., 2020)). A vane pump (135 FZ 3 V, Schwarz Precision, Germany) provided the flow

114 for sampling and flushing to recover the separation column.

115 The gas sensor consists of Pd-doped $\mathrm{SnO}_{2}$ nanoparticles made by flame spray pyrolysis

116 and directly deposited onto micromachined sensor substrate (Güntner et al., 2016) (1.9×1.7 mm²,

117 MSGS 5000i, Microsens SA, Switzerland) featuring interdigitated electrodes and a heater on a

118 free-standing membrane. This sensor was mounted onto a leadless chip carrier (LCC, Chelsea 
119 Technology Inc., U.S.A.) with high temperature carbon paste (Ted Pella Inc., U.S.A.) and

120 electrically connected through aluminum wires $(30 \mu \mathrm{m}$ in diameter) by bonding (F\&K Delvotec,

121 Germany). After placing it on a socket (E-Tec, Switzerland) that was soldered to a printed circuit

122 board (PCB), the sensor was sealed (gas-tight) by an inert Teflon chamber with its design

123 disclosed elsewhere (Abegg, et al., 2020). A microcontroller (Raspberry pi Zero W, U.S.A.)

124 provided the required heating power to operate the sensor at $350{ }^{\circ} \mathrm{C}$ (van den Broek, et al.,

125 2019), monitored its resistance and communicated data wirelessly to a smartphone by Bluetooth

126 or Wi-Fi. The smartphone prototype app was made with a free mobile app constructor (Version

127 2.27.19, Blynk Inc., U.S.A.).

\section{Sample preparation}

129 The applied substances were methanol (> 99.9\%, Sigma-Aldrich, Germany), ethanol (> 99.8\%,

130 Fisher Chemical, Switzerland), 1-propanol (> 99\%, Merck, Germany), 2-propanol (> 99.5\%,

131 Sigma Aldrich, Germany), butanone (> 99\%, VWR International, France) and Milli-Q water

132 (Milli-Q Synthesis A10, Merck, Germany). Also seven commercial hand sanitizers were tested

133 with their identifiers, producers and compositions, as available, listed in Table 1. Binary, ternary

134 (for calibration) mixtures and methanol-spiked hand sanitizers were obtained by admixing the

135 desired amounts of methanol with high precision pipettes. Each sample was $5 \mathrm{~mL}$ prepared in 20

136 mL glass vials (Vial SCR 20ML, VWR, Germany) leaving sufficient headspace for vapor

137 analysis. The vials were sealed immediately after preparation with caps (polypropylene screw

138 cap with hole $24 \mathrm{~mm}$, Supelco, U.S.A.) containing a septum (Teflon faced silicone septa $22 \mathrm{~mm}$,

139 Supelco, U.S.A.), unless otherwise stated. 


\section{Headspace analysis}

141 Right before each sensor measurement, the prepared vials were rigorously shaken (at least $30 \mathrm{~s}$ )

142 to afford phase equilibrium in the vial (Abegg, et al., 2020). Next, the capillary of the detector

143 was inserted through the vial septum together with a second capillary for pressure balance. Note

144 that sampling can be done also from the open container (Figure 1), though this is less accurate

145 (Figure S-5) due to higher dilution with surrounding air. Sample was extracted always for $10 \mathrm{~s}$ at

146 a sampling rate of $25 \mathrm{~mL} \mathrm{~min}^{-1}$ drawn by the vane pump. Afterwards the capillary was removed

147 from the vial and ambient air was drawn continuously to transport the sample through the

148 separation column and to the sensor. By flushing with ambient air at $65 \mathrm{~mL} \mathrm{~min}^{-1}$, residual

149 adsorbate was removed from the separation column to facilitate fast detector reusability. After

150 recovery, the flow rate was set to zero to reduce the amount of noise due to ambient air

151 interferants (Abegg, et al., 2020).

152 The sensor response (S) was defined as:

(1) $S=\frac{R_{b}}{R_{S}}-1$

154 with $\mathrm{R}_{\mathrm{b}}$ and $\mathrm{R}_{\mathrm{s}}$ being the sensor (i.e. Pd-doped $\mathrm{SnO}_{2}$ film) resistances at baseline (stabilized in

155 room air) and under sample exposure, respectively. The $t_{R}$ of an analyte was defined as the time

156 required to reach the response peak, similar to gas chromatography (Geankoplis, 2003). The

157 methanol concentration in pure and spiked hand sanitizers were quantified by comparing the

158 peak response to five-point calibration curves from methanol-ethanol-water mixtures (giving

159 similar methanol responses to mixtures with 2-propanol instead of ethanol, Figure 2c) in the

160 expected concentration range, as elaborated elsewhere (Abegg, et al., 2020).

161 The methanol content of pure and spiked hand sanitizers \#1-6 was determined also by gas

162 chromatography for comparison. Note that gel-type hand sanitizer \#7 was not analyzed due to its

163 high viscosity. Measurements were performed on a Varian 3800 (Agilent, U.S.A.) with a column 
164 (Zebron ZB-624, Brechbühler AG, Switzerland) and flame ionization detector operated at 45 and

$165220{ }^{\circ} \mathrm{C}$, respectively. The sampling volume and pressure were $0.5 \mu \mathrm{L}$ and 4 psi, respectively and

166 the injector was applied at $210{ }^{\circ} \mathrm{C}$ with split ratio 20 . Methanol concentrations were obtained by

167 comparing the area under curve of the methanol signal to calibration curves, as evaluated with

168 the software Varian Star Chromatography Workstation (Agilent, U.S.A.). The calibration was

169 done with the above-mentioned standards by mixing the desired amounts with precision

170 graduated and volumetric pipettes (Hirschmann, Germany) in a $100 \mathrm{~mL}$ volumetric flask and

171 analyzing the peak response area (McNair et al., 2019).

\section{Results and Discussion}

\section{Analytical strategy}

174 The handheld device is shown in Figure 1. For hand sanitizer analysis, headspace vapor is

175 extracted for $10 \mathrm{~s}$ through a sampling capillary with a vane pump. When transported through the

176 separation column (i.e. packed bed of non-polar Tenax TA polymer particles), the analytes are

177 separated by sorption (similar to gas chromatography) on the Tenax TA available surface area

178 (van den Broek, et al., 2019) of $35 \mathrm{~m}^{2} \mathrm{~g}^{-1}$. Specifically, larger alcohols (e.g. ethanol, 2-propanol),

179 the main constituents of hand sanitizers (Table 1), are retained longer than methanol due to

180 stronger van-der-Waals adsorption forces (Maier and Fieber, 1988) rendering the device

181 selective. This represents a key challenge for conventional chemical sensors that can hardly

182 distinguish these molecules (Guo, et al., 2011) due to their chemical similarity (i.e. hydroxyl

183 group).

184 A chemoresistive micro-gas sensor upstream the separation column detects and quantifies

185 the methanol content. It is based on a porous film, self-assembled by flame-aerosol deposition of

$186 \mathrm{SnO}_{2}$ nanoparticles (grain size $16 \mathrm{~nm}$ (Abegg, et al., 2020)) containing lattice-incorporated and 
187 surface-loaded Pd (Pineau et al., 2020) that feature high sensitivity to various volatile organics

188 (e.g. down to 3 ppb formaldehyde at 90\% relative humidity (Güntner, et al., 2016)). Methanol is

189 adsorbed on these nanoparticles (Ouyang et al., 2000) and converted by chemical reaction with

190 oxygen- and hydroxyl-related species (Cheong and Lee, 2006). The associated release of

191 electrons into the n-type semiconducting $\mathrm{SnO}_{2}$ results in a measurable signal (i.e. film resistance

192 change) (Ogawa et al., 1982) that is proportional to methanol concentration. All other parts of the

193 device in contact with analytes (e.g. tubing, sensor housing, etc.) are made of inert Teflon to

194 minimize adsorption and contamination. After flushing the column and sensor with ambient air

195 to remove residual adsorbate, it can be reused after $15 \mathrm{~min}$ and provides stable results tested

196 during more than three months (Abegg, et al., 2020).

197 Selective methanol detection over other alcohols

198 Figure 2a shows the sensor response curves for $0-100$ vol\% methanol in ethanol. Methanol

199 passes through the separation column first with retention times $\left(t_{R}\right)$ between $1.5-0.8$ min for

$2000.01-100 \mathrm{vol} \%$, respectively, in agreement with literature (i.e. $1.25 \mathrm{~min}$ for $10 \mathrm{vol} \%$ methanol

201 in 80 vol\% ethanol and water (Abegg, et al., 2020)). Note that shorter retention times with

202 increasing methanol levels are due to an overloading of the column, as with gas chromatography

203 (Yabumoto et al., 1980), but this does not affect methanol quantification, as shown below. Most

204 importantly, ethanol elutes later $\left(t_{R}=2 \mathrm{~min}\right.$ for pure ethanol, Figure S-1) without interfering the

205 methanol measurement. Similarly, 2-propanol (Figure 2b) passes the separation column even

206 later $\left(t_{R}=2.8\right.$ min for pure 2-propanol, Figure $\left.S-1\right)$ with rather small response. As a result,

207 methanol is detected selectively over all alcohols overcoming a major bottleneck in chemical

208 sensing. 
Another challenge is the quantification of methanol over a large concentration range: at

210 least from 0.063 vol\% (U.S. Food and Drug Administration, 2020b) (FDA limit) to 81 vol\%

211 (max. content found in adulterated sanitizers (U.S. Food and Drug Administration, 2020a)). This

212 is met by the device that detects methanol over four orders of magnitude $(0.01-100$ vol\%,

213 Figure $2 \mathrm{c}$ ) with almost identical responses (average deviation $4 \%, \mathrm{R}^{2}=0.99$ ) in ethanol (squares)

214 and 2-propanol (circles), highlighting again its excellent selectivity. Remarkably, even lowest

2150.01 vol\% (Insets, Figure 2a and Figure 2b) are detected with high signal-to-noise (SNR > 300)

216 within $2 \mathrm{~min}$ at very high alcohol background (i.e. $>99$ vol\%). The recognition of such low

217 methanol concentrations is superior to state-the-art sensors featuring higher detection limits, for

218 instance, electrochemical cells (Ou, et al., 2019) (0.15 vol\%) or fluorescent sensors (Huang et al.,

219 2018) (4 vol\%). Also close to the FDA limit, methanol concentrations are distinguished clearly,

220 as demonstrated for 0.05, 0.06 and 0.07 vol\% (Insets, Figure 2a and Figure 2b). Please note that

221 the $t_{R}$ at such low methanol concentrations are slightly higher (e.g. 1.6 vs. 1.5 min at 0.06 vol\%)

222 in 2-propanol than ethanol, probably due to competitive adsorption (Comes et al., 1993) on the

223 Tenax TA and the higher vapor pressure of ethanol.

\section{Hand sanitizers}

225 Hand sanitizers are typically more complex mixtures containing also humectants, odorants,

226 denaturants and colorants. Thus, the device was evaluated (Figure 3a) on six commercially

227 available hand sanitizers with different compositions (Table 1), as characterized also by gas

228 chromatography (Figure S-2). Sanitizers \#1 - 5 are ethanol-based, as correctly recognized by the

229 device. On the other hand, hand sanitizer \#6 contains mainly 2- (49 vol\%) and 1-propanol (32

230 vol\%) with both compounds being identified by the sensor (Figure S-3). It should be noted that

231 the FDA considers 1-propanol toxic (U.S. Food and Drug Administration, 2020b) and has 
232 limited its content also to 0.1 vol $\%$ while it is recommended as active substance in biocidal

233 products in the E.U. (European Chemical Agency, 2020).

234 Only sample \#2 contained methanol, as detected by the device with a response of 2.2 at

$235 \quad\left(t_{R}\right) 1.4$ min and confirmed by gas chromatography (0.19 vol\%, Figure $\left.\mathrm{S}-2\right)$. This hand sanitizer

236 is based on fruit-derived distillates where methanol is formed naturally during fermentation

237 (from pectin degradation (Bindler et al., 1988)). Please note that its methanol content, however,

238 is below the E.U. limit (i.e. 0.9 vol\% at that ethanol content (European Parliament and Council,

239 2019)) for fruit distillates.

240 Next, these hand sanitizers were spiked with $0.01-90$ vol\% methanol (total 66 samples)

241 to simulate the entire range of typical contamination/adulteration. Figure $3 \mathrm{~b}$ shows the sensor

242 response exemplarily for sample \#5 that contains 81 vol\% ethanol (Table 1) but also glycerol,

243 panthenol, cyclopentasiloxane, cyclohexasiloxane, isotrideceth-8, 2-propanol, and

244 didecyldimethylammoniumchloride (please see Figure S-4 for sample \#3). Remarkably, these

245 compounds do not interfere the measurement. In fact, methanol elutes at comparable $t_{\mathrm{R}}$ to the

246 binary mixtures with ethanol (Figure 2a) and is quantified with similar response (1.5 vs. 1.7 for

2470.1 vol\% methanol). We confirmed this also through experiments with pure substances (Figure

248 S-1) where other compounds were detected only after 2 min being higher than the methanol $t_{R}$

249 for lowest $0.01 \mathrm{vol} \%$ (i.e. $1.5 \mathrm{~min}$ ).

250 Figure $3 \mathrm{c}$ shows the methanol concentrations of pure and spiked hand sanitizers, as

251 measured by our detector and "gold standard" gas chromatography. The detector quantifies

252 methanol accurately over four orders of magnitude with high $\mathrm{R}^{2}$ of 0.99 . The error is fairly small

253 (95\% confidence interval: -18.5 to $16.4 \%$, dashed lines in Figure 3b) and stays rather constant

254 over the entire measurement range, as revealed by Bland-Altman analysis (Martin Bland and 
255 Altman, 1986). In other words, methanol concentrations at the FDA limit (0.063 vol\%) will be 256 determined between $0.051-0.073 \mathrm{vol} \%$, which should be sufficiently accurate for screening

257 hand sanitizers. Consequently, methanol is detected reliably in the commercial hand sanitizers

258 \#1-6 despite their different compositions (Table 1). Also colorants (e.g. \#6 contains patent blue

$259 \mathrm{~V}$ ) do not interfere the measurement (Figure 3c, inverse triangles), that may be quite problematic

260 for colorimetric tests (e.g. Alert for Methanol).

261 Finally, we tested also the gel-like hand sanitizer \#7 (Figure 4) to assess viscosity effects.

262 Most importantly, the spiked methanol concentrations were recognized well with high $\mathrm{R}^{2}(0.99)$,

263 consistent to the less viscous samples \#1 - 6 (Figure 3c). This highlights the robustness of present

264 headspace analysis even for highly viscous samples where commercial colorimetric assays might

265 fail, as indicator solutions do not mix well with such fluids.

266 We anticipate this device to be helpful to police, customs, distributors and consumers to

267 check product safety. It is compact $\left(2 \times 4 \times 12 \mathrm{~cm}^{3}\right.$, Figure 1$)$, weighs only $94 \mathrm{~g}$ and offers low

268 power consumption (ca. 1.1 W during analysis) enabling battery-driven operation(Abegg, et al.,

269 2020). The operation and data display are user-friendly by providing wireless communication by

270 Wi-Fi or Bluetooth, functioning even if no external network is available. When combined with a

271 breath sampler, this device is even applicable for medical screening of methanol poisoning by

272 breath analysis (van den Broek, et al., 2019), as established for ethanol by law enforcement

273 (Güntner et al., 2019).

\section{Conclusions}

275 We presented a handheld and readily applicable detector for distributed and on-site screening of

276 sanitizers for toxic methanol. It quantifies methanol within two minutes selectively over four

277 orders of magnitude $(0.01-100 \mathrm{vol} \%)$ and meets even newest national guidelines (e.g. FDA), as 
278 validated by gas chromatography. Typical hand sanitizer constituents and gel-like viscosity do

279 not interfere the measurement while other potential contaminants (e.g. 1-propanol) are

280 recognized as well. The device operation and data analysis is user-friendly, providing results on

281 smartphones where further communication to data clouds for remote analysis is possible. The

282 device contains mostly commercially available components, thus can be produced at low cost

283 and large numbers. It addresses an urgent need during the COVID-19 health crisis where

284 widespread access to safe sanitizers is crucial to mitigate disease propagation.

\section{CRediT authorship contribution statement}

286 Andreas T. Güntner: Conceptualization, Methodology, Investigation, Visualization, Writing -

287 Original Draft, Project administration, Funding acquisition. Leandro Magro: Conceptualization,

288 Methodology, Investigation, Writing - Review \& Editing. Jan van den Broek:

289 Conceptualization, Methodology, Investigation, Writing - Review \& Editing. Sotiris E.

290 Pratsinis: Conceptualization, Writing - Review \& Editing, Project administration, Funding

291 acquisition.

\section{Acknowledgments}

293 This project was supported by the Particle Technology Laboratory ETH Zürich and, in part, by

294 the ETH Zürich Research Commission (Project ETH-05 19-2) and the Swiss National Science

295 Foundation (grant 175754 and R'EQUIP 170729). We thank M. Mazzotti (ETH Zurich) for

296 providing access to gas chromatography.

\section{Declaration of Competing Interests}

298 A patent application for this methanol detector has been submitted by ETH Zürich. 


\section{Supporting Information}

300 Sensor responses to sanitizer-related pure substances (Figure S-1); gas chromatograms of pure 301 commercial hand sanitizers and reference substances (Figure S-2); full sensor response to pure 302 sanitizer \#6 (Figure S-3); sensor responses of pure and methanol-spiked sanitizer \#3 (Figure S303 4); detector sampling with sealed and open vials (Figure S-5).

\section{References}

Abegg, S., Magro, L., van den Broek, J., Pratsinis, S.E., Güntner, A.T., 2020. A pocket-sized device enables detection of methanol adulteration in alcoholic beverages. Nature Food 1, 351-354.

Barceloux, D.G., Randall Bond, G., Krenzelok, E.P., Cooper, H., Allister Vale, J., 2002. American Academy of Clinical Toxicology practice guidelines on the treatment of methanol poisoning. J. Toxicol., Clin. Toxicol. 40, 415-446.

Bindler, F., Voges, E., Laugel, P., 1988. The problem of methanol concentration admissible in distilled fruit spirits. Food Addit. Contam. 5, 343-351.

Cheong, H., Lee, M., 2006. Sensing characteristics and surface reaction mechanism of alcohol sensors based on doped $\mathrm{SnO}_{2}$. J. Ceram. Process. Res. 7, 183.

Comes, P., Gonzalez-Flesca, N., Menard, T., Grimalt, J.O., 1993. Langmuir-derived equations for the prediction of solid adsorbent breakthrough volumes of volatile organic compounds in atmospheric emission effluents. Anal. Chem. 65, 1048-1053.

Dicken, R.D., Gallagher, T., Perks, S., 2020. Overcoming the Regulatory Hurdles for the Production of Hand Sanitizer for Public Health Protection: The UK and US Academic Perspective. ACS Chem. Health Saf. 27, 209-213.

European Chemical Agency, 2020. Recommended requirements for the active substances Propan-1-ol and Propan-2-ol, for the purpose of derogations under Article 55(1) of the BPR.

European Parliament and Council, 2019. Regulation (EU) No 2019/787. Official Journal of the European Union

Fazio, M., 2020. 3 Die in New Mexico After Drinking Hand Sanitizer, Officials Say. The New York Times

Geankoplis, C.J., Transport processes and separation process principles:(includes unit operations), Prentice Hall Professional Technical Reference, 2003.

Government of Canada, 2020. Recall of certain hand sanitizers that may pose health risks, https://healthycanadians.gc.ca/recall-alert-rappel-avis/hc-sc/2020/73385a-eng.php. 
Güntner, A.T., Koren, V., Chikkadi, K., Righettoni, M., Pratsinis, S.E., 2016. E-Nose Sensing of Low-ppb Formaldehyde in Gas Mixtures at High Relative Humidity for Breath Screening of Lung Cancer? ACS Sens 1, 528-535.

Güntner, A.T., Abegg, S., Konigstein, K., Gerber, P.A., Schmidt-Trucksass, A., Pratsinis, S.E., 2019. Breath Sensors for Health Monitoring. ACS Sens 4, 268-280.

Güntner, A.T., Wied, M., Pineau, N.J., Pratsinis, S.E., 2020. Rapid and Selective $\mathrm{NH}_{3}$ Sensing by Porous CuBr. Adv. Sci. 7, 1903390.

Guo, H., Chen, X., Yao, Y., Du, G., Li, H., 2011. Detection of ethanol and methanol vapors using polymer-coated piezoresistive Si bridge. Sens. Actuators B 155, 519-523.

Huang, R., Liu, K., Liu, H., Wang, G., Liu, T., Miao, R., Peng, H., Fang, Y., 2018. Film-Based Fluorescent Sensor for Monitoring Ethanol-Water-Mixture Composition via Vapor Sampling. Anal. Chem. 90, 14088-14093.

Kampf, G., Todt, D., Pfaender, S., Steinmann, E., 2020. Persistence of coronaviruses on inanimate surfaces and their inactivation with biocidal agents. J. Hosp. Infect. 104, 246251.

Kraut, J.A., Kurtz, I., 2008. Toxic Alcohol Ingestions: Clinical Features, Diagnosis, and Management. Clin. J. Am. Soc. Nephrol. 3, 208-225.

Kraut, J.A., Mullins, M.E., 2018. Toxic Alcohols. New Engl. J. Med. 378, 270-280.

Maier, I., Fieber, M., 1988. Retention characteristics of volatile compounds on tenax TA. J. High. Resolut. Chromatogr. 11, 566-576.

Martin Bland, J., Altman, D., 1986. Statistical Methods for Assessing Agreement between Two Methods of Clinical Measurement. Lancet 327, 307-310.

McNair, H.M., Miller, J.M., Snow, N.H., Basic gas chromatography, John Wiley \& Sons, 2019.

Meng, D., Zhang, S., Thomas, T., Huang, C., Zhao, J., Zhao, R., Shi, Y., Qu, F., Yang, M., 2020. Pt/WN based fuel cell type methanol sensor. Sens. Actuators B 307, 127686.

Ogawa, H., Nishikawa, M., Abe, A., 1982. Hall measurement studies and an electrical conduction model of tin oxide ultrafine particle films. J. Appl. Phys. 53, 4448-4455.

Ou, T.-H., Wang, Y., Fang, D., Narayanan, S., Wu, W., 2019. Detection of Fake Alcoholic Beverages Using Electrolyte-Free Nanogap Electrochemical Cells. ACS Appl. Mater. Interfaces 11, 6217-6223.

Ouyang, F., Yao, S., Tabata, K., Suzuki, E., 2000. Infrared identification of reaction route from adsorbed species derived from adsorption of methanol on $\mathrm{SnO}_{2}$. Appl. Surf. Sci. 158, 2831.

Park, C.H., Koo, W.-T., Lee, Y.J., Kim, Y.H., Lee, J., Jang, J.-S., Yun, H., Kim, I.-D., Kim, B.J., 2020. Hydrogen Sensors Based on $\mathrm{MoS}_{2}$ Hollow Architectures Assembled by Pickering Emulsion. ACS Nano DOI: 10.1021/acsnano.1020c00821.

Pineau, N.J., Keller, S.D., Güntner, A.T., Pratsinis, S.E., 2020. Palladium embedded in SnO2 enhances the sensitivity of flame-made chemoresistive gas sensors. Microchim Acta 187, 96. 
U.S. Department of Defense, 2020. Global CBRN Detector Market Survey, https://www.wmddetectorselector.army.mil/library/Global\%20CBRN\%20Detector\%20Ma rket\%20Surveypage_final_sm.pdf.

U.S. Food and Drug Administration, 2020b. Temporary policy for preparation of certain alcoholbased hand sanitizer products during the public health emergency (COVID-19): Guidance for industry.

U.S. Food and Drug Administration, 2020a. FDA updates on hand sanitizers consumers should not use, https://www.fda.gov/drugs/drug-safety-and-availability/fda-updates-handsanitizers-consumers-should-not-use.

van den Broek, J., Abegg, S., Pratsinis, S.E., Güntner, A.T., 2019. Highly selective detection of methanol over ethanol by a handheld gas sensor. Nature Commun. 10, 4220.

van den Broek, J., Cerrejon, D.K., Pratsinis, S.E., Güntner, A.T., 2020. Selective formaldehyde detection at ppb in indoor air with a portable sensor. J. Hazard. Mater. 123052.

Wambua-Soi, C., 2020. Over 700 dead after drinking alcohol to cure coronavirus. Al-Jazeera

Weber, I.C., Braun, H.P., Krumeich, F., Güntner, A.T., Pratsinis, S.E., 2020. Superior Acetone Selectivity in Gas Mixtures by Catalyst-Filtered Chemoresistive Sensors. Adv. Sci. 2001503, DOI: 2001510.2001002/advs.202001503.

World Health Organization, Guide to local production: WHO-recommended handrub formulations, in, 2010.

Wu, F., Zhao, S., Yu, B., Chen, Y.-M., Wang, W., Song, Z.-G., Hu, Y., Tao, Z.-W., Tian, J.-H., Pei, Y.-Y., Yuan, M.-L., Zhang, Y.-L., Dai, F.-H., Liu, Y., Wang, Q.-M., Zheng, J.-J., Xu, L., Holmes, E.C., Zhang, Y.-Z., 2020. A new coronavirus associated with human respiratory disease in China. Nature 579, 265-269.

Yabumoto, K., Ingraham, D.F., Jennings, W.G., 1980. The overload phenomenon in gas chromatography. J. High. Resolut. Chromatogr. 3, 248-252.

Zhao, H., Rizal, B., McMahon, G., Wang, H., Dhakal, P., Kirkpatrick, T., Ren, Z., Chiles, T.C., Naughton, M.J., Cai, D., 2012. Ultrasensitive Chemical Detection Using a Nanocoax Sensor. ACS Nano 6, 3171-3178. 
401 Table 1. Analyzed commercial hand sanitizers and their composition, as indicated by supplier.

402 Contents by volume are indicated in brackets, if available.

\begin{tabular}{|l|l|l|}
\hline Brand & Sample & Composition (vol \%) \\
\hline B. Braun Medical & $\# 1$ & Ethanol (85), glycerol (0.7), butanone (<3) \\
\hline *WHO & $\# 2$ & $\begin{array}{l}\text { Ethanol (72), glycerol (1.45), hydrogen peroxide } \\
(0.125), \text { rest water }\end{array}$ \\
\hline Martec Desinfektion & $\# 3$ & Ethanol (82) \\
\hline $\begin{array}{l}\text { Lactipar Desin } \\
\text { Händedesinfektion } \\
\text { Händedesinfektionsmittel }\end{array}$ & $\# 4$ & Ethanol (>80), butanone (<5.3) \\
\hline Sterillium & $\# 6$ & $\begin{array}{l}\text { Alcohol denat. (81), water, glycerol, panthenol, } \\
\text { cyclopentasiloxane, cyclohexasiloxane, } \\
\text { isotrideceth-8, 2-propanol, } \\
\text { didecyldimethylammoniumchloride (0.05 vol\%) }\end{array}$ \\
\hline $\begin{array}{l}\text { Martec Hand- } \\
\text { Desinfektion Gel }\end{array}$ & $\# 7$ (gel) & $\begin{array}{l}\text { 2-propanol (49), 1-propanol (32) } \\
\text { mecetroniumetilsulfat (0.2), glycerol, } \\
\text { tertradecanol, odorants, patent blue V, water }\end{array}$ \\
\hline
\end{tabular}

403

404

405

406

*Mixed according to WHO hand rub formulation (World Health Organization, 2010) but with fruit spirit-derived ethanol. 


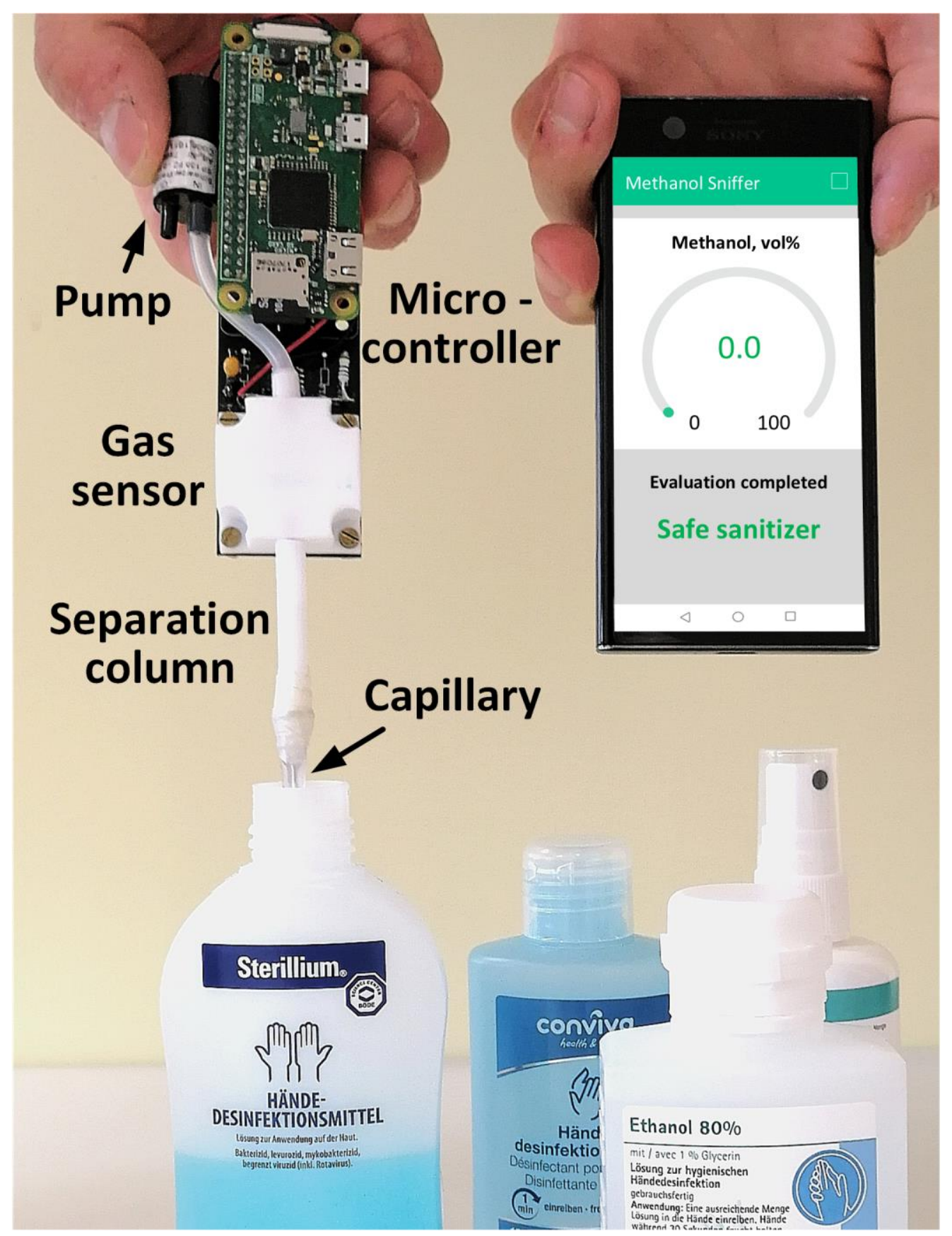

408 Figure 1. Handheld methanol detector for screening hand sanitizers. Key components are the 409 capillary for vapor sampling, separation column, gas sensor (sealed by chamber), pump and 410 micro-controller. Data is communicated wirelessly to a smartphone and an exemplary user411 interface is shown. 

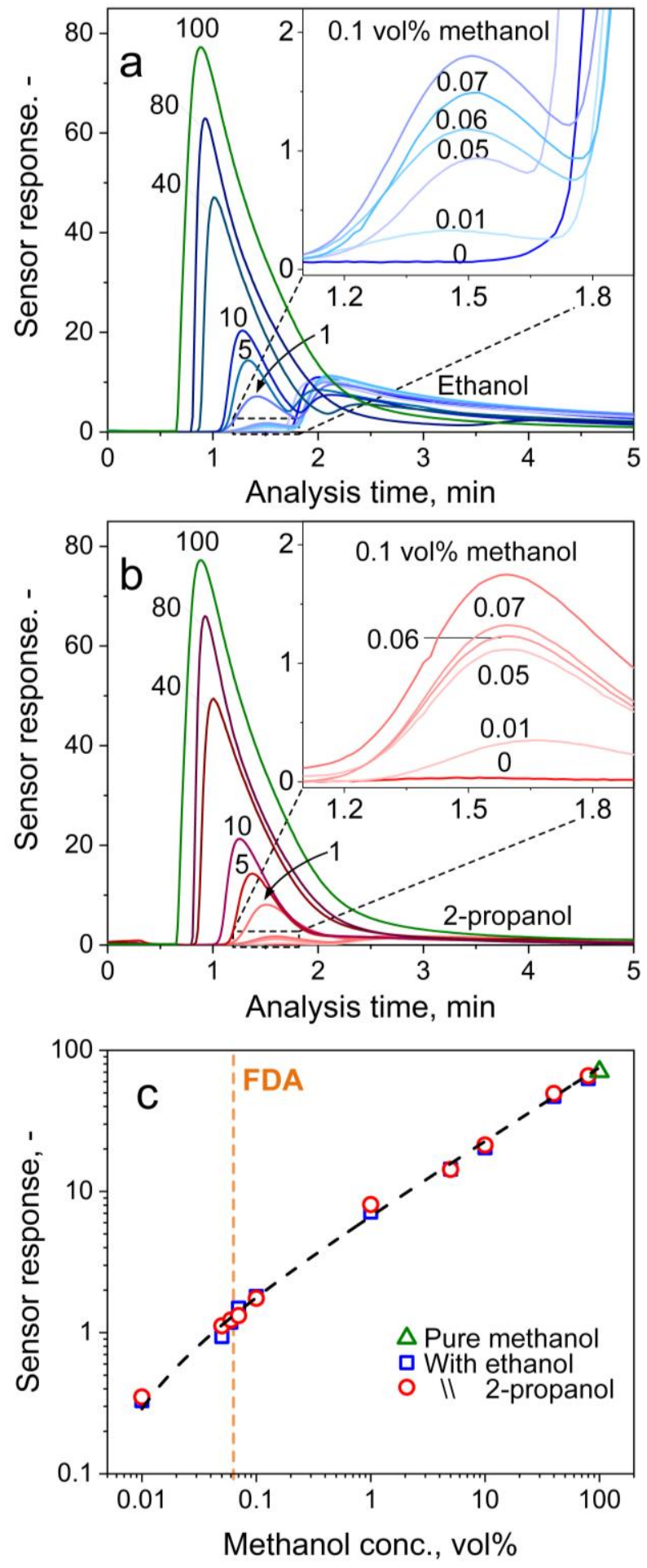

413 Figure 2. Sensor response to $0-100$ vol\% methanol in ethanol (a) or 2-propanol (b). Insets 414 magnify $0-0.1$ vol\% methanol. (c) Sensor response peak values for pure methanol (triangle) 415 and with ethanol (squares) or 2-propanol (circles). Indicated is also the FDA recommended limit 416 (i.e. 0.063 vol\%, vertical dashed line) and best fit (black dashed line). 

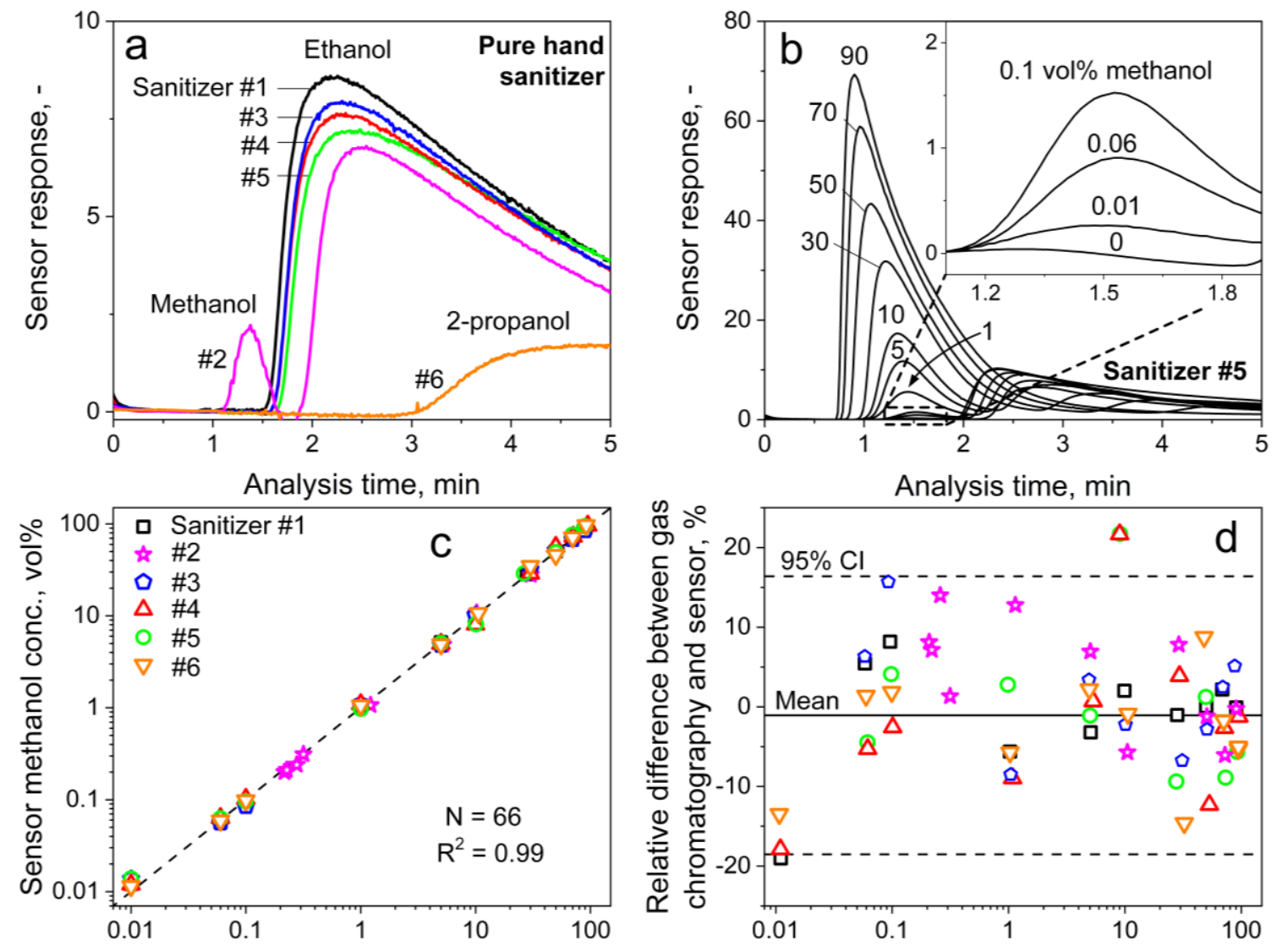

Average of gas chromatography and sensor, vol\%

418 Figure 3. Commercial hand sanitizers \#1-6 (pure and methanol-spiked) evaluated by sensor and

419 gas chromatography: (a) Sensor response to the commercial hand sanitizers with different

420 compositions (Table 1). Associated peaks for methanol, ethanol and 2-propanol are indicated. (b)

421 Response to 0 - 90 vol\% methanol-spiked samples of sanitizer \#5 that contains 81 vol\% ethanol,

422 water, glycerol, panthenol, cyclopentasiloxane, cyclohexasiloxane, isotrideceth-8, 2-propanol

423 and didecyldimethylammoniumchloride (Table 1). Inset shows magnification of $0-0.1 \mathrm{vol} \%$

424 methanol content. (c) Scatter plot (66 samples) indicating the methanol content in pure and

425 spiked hand sanitizers, as measured by sensor and gas chromatography. (d) Corresponding

426 Bland-Altman analysis indicating the relative error of the measured methanol concentrations vs.

427 the average concentration measured by both instruments. Mean and limits of agreement $(95 \%$

428 confidence intervals, CI) are provided as solid and dashed lines, respectively. 


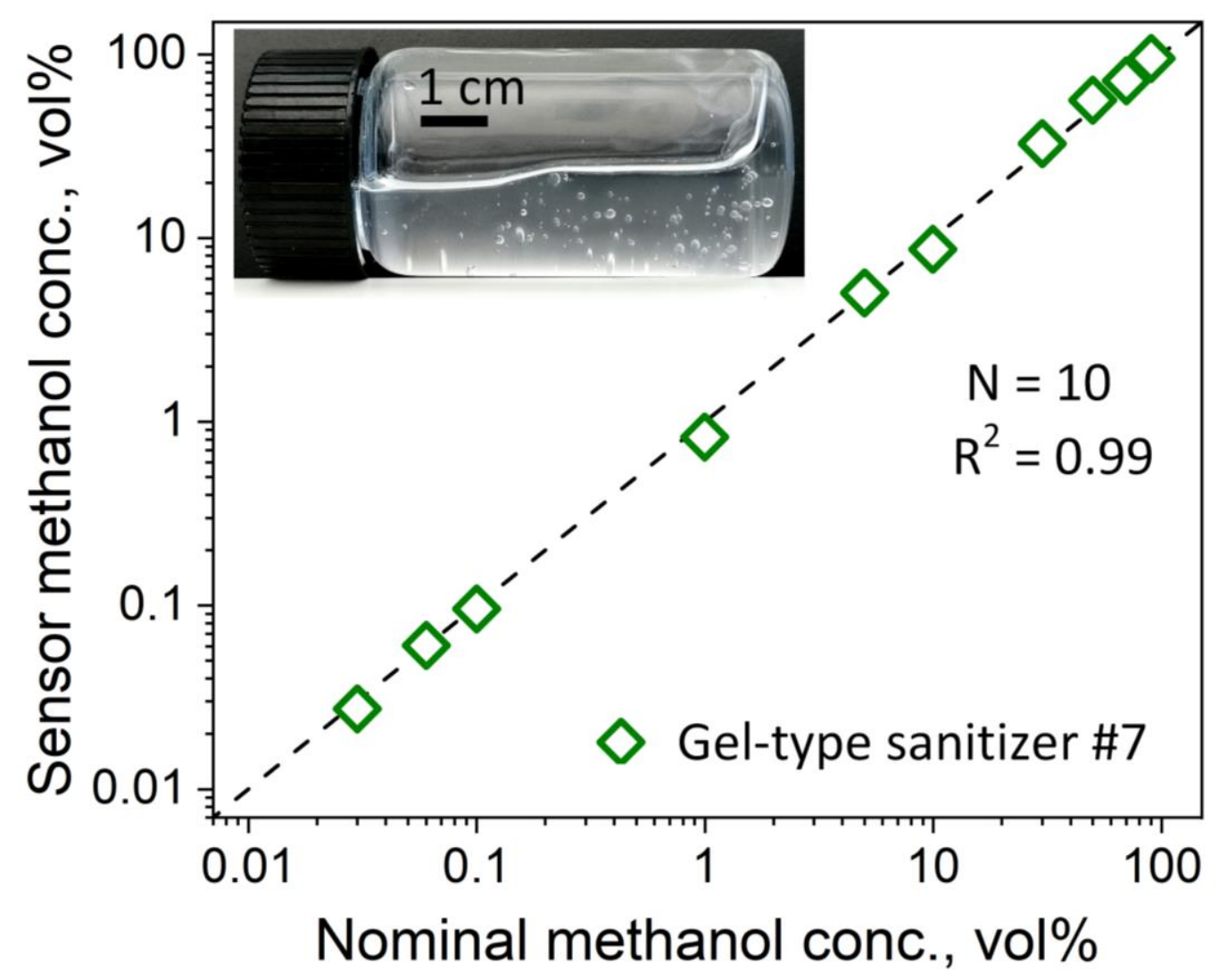

430 Figure 4. Methanol concentration measured by the sensor in gel-like hand sanitizer \#7

431 (methanol-spiked). Note that direct analysis by gas chromatography was not feasible due to the 432 sanitizer's high viscosity. Inset shows the sample. 